\title{
Monte Carlo Computer Simulation in Horticulture: A Model for Container Media Characterization
}

\author{
Silvia Burés \\ Department of Horticulture and Center for Simulational Physics, University of Georgia, Athens, GA 30602-7273 \\ David P. Landau and Alan M. Ferrenberg ${ }^{1}$ \\ Center for Simulational Physics, University of Georgia, Athens, GA 30602-2451 \\ Franklin A. Pokorny \\ Department of Horticulture, University of Georgia, Athens, GA 30602-7273
}

The Monte Carlo method of computer simulation is a powerful tool for studying physical systems. With this method, random numbers are used to simulate processes that occur randomly in nature (Gould and Tobochnik, 1988). Monte Carlo methods are used in such diverse research areas as physics and materials science, economics, medicine, biochemistry, communications, and other fields. Computer simulation permits one to ascertain the quality of models for processes; it is flexible and allows precise control of various variables, facilitating the study of multicomponent systems, parallel and interrelated phenomena, or long-term variation processes (Binder, 1986). There are multiple advantages to using simulation: it can save time, labor, and money, compared with traditional laboratory experiments, while eliminating the safety hazards that may be encountered when conducting experiments (Hammersley and Handscomb, 1964). Many phenomena in horticulture take place at random; one of them is the container medium that constitutes the skeleton of the rhizosphere.

Packings of spheres serve as models for many physical systems (Barker and Grimson, 1990; Dallavalle, 1948; Finney, 1983) and are achieved by establishing particle coordinates that represent a real assembly of particles constituting a structured system from which several properties can be calculated (Finney, 1983).

A container medium can be considered a random system of particles. Applying simulation techniques to container medium systems is contingent on simultaneously characterizing their packing patterns and establishing analogies between container media and geometrical particles. This approach has long been considered useful in studying soil properties (Slichter, 1899).

New materials that might be useful in container medium formulation are becoming avail-

\footnotetext{
Received for publication 25 Mar. 1993. Accepted for publication 26 Mar. 1993. Supported by state and Hatch funds allocated to the Georgia Experiment Station. We acknowledge Steven E. Follin (Univ. Computing and Networking Services) for his assistance on graphical visualization of our data. The cost of publishing this paper was defrayed in part by the payment of page charges. Under postal regulations, this paper therefore must be hereby marked advertisement solely to indicate this fact. ${ }^{1}$ Present address: Univ. Computing and Networking Services, Univ. of Georgia, Athens, GA 30602-1911.
}

able because of the need to recycle industrial and agricultural by-products (Penninck et al., 1985). Because container media consist of mixtures of various materials in diverse proportions, laboratory and field evaluations are often complex and time consuming. Some researchers have attempted to model container media (Burés et al., 1988; okorny, 1981) and to establish correlations between various container media properties (Bugbee and Frink, 1983; Milks et al., 1989), and between soil properties and plant growth (Tilt et al., 1987).

Monte Carlo simulation of random systems of particles, when applied to container media, would provide a means of understanding those characteristics that are related to particle packing. Modeling the characteristics of container media serves our aim to improve accessibility of Monte Carlo simulation to horticulturists.

\section{THE APPROACH}

\section{Monte Carlo method}

The Monte Carlo method is used in statistical physics to study models of thermodynamic systems by computer simulation (Binder, 1986; Ferrenberg and Landau, 1991; Landau and Alben, 1973). A simple introduction to the method can be found in Gould and Tobochnik (1988). With the Monte Carlo method, particles are placed in some configuration inside a container. Then an attempt is made to move each particle in succession to a new position in the system within some maximum allowed displacement. The change in the system's energy that would occur if the particle were moved to the new position is calculated. If the energy decreases, then the particle is moved to the new position; if the energy increases, then the particle is moved only with a certain probability. We have implemented an algorithm based on the "importance sampling" method of Metropolis et al. (1953), where the probability of moves that increase the energy depends on the system's temperature. One Monte Carlo step is an attempt to move each particle in the system.

Each particle in a system is labeled by its y and $z$ coordinates, $\overrightarrow{\mathbf{r}}_{j}=\left(\mathrm{y}_{\mathrm{j}}, \mathrm{z}_{\mathrm{j}}\right)$ We have only two coordinates because we are working in two dimensions. The system's configuration is designated by giving the coordinates of every particle: $\overrightarrow{\mathbf{R}}=\left\{\overrightarrow{\mathbf{r}}_{1} \ldots \overrightarrow{\mathbf{r}}_{\mathrm{N}}\right\}$ To perform a Monte Carlo simulation, one must specify how the particle's energy depends on its location.

In our model, the energy in a configuration is the gravitational potential energy:

$$
E(\vec{R})=\sum_{j=1}^{N} m_{j} \times g \times z_{j}
$$

where $m_{j}$ is the mass of the particle $j, g$ is the gravitational constant, and $\mathrm{z}_{\mathrm{j}}$ is the height of particle j. $E(\vec{R})$ s the gravitational potential energy of the system. The probability for a given configuration, $P[\mathrm{E}(\overrightarrow{\mathrm{R}})$ is made proportional to exp $[-\mathrm{E}(\overrightarrow{\mathrm{R}}) / \mathrm{k} \times \mathrm{T}$ ith the "importance sampling" method, where $\mathrm{k}$ is the Boltzmann constant and T is the system's absolute temperature. In our case, we are using the temperature to establish an analogy between our particular system and a thermodynamic system. Once the energy of the system is defined, we attempt to move each particle in succession, with length and direction of displacement chosen randomly. No particle overlap is allowed; therefore, a move that would result in overlapping is rejected directly. The change in energy, $\Delta \mathrm{Ej}$, caused by an allowed move is then calculated. The trial configuration is accepted with $P=1$ (always accepted) if the energy decreases, and if the energy increases, this move is allowed with $P$ :

$$
P\left[\Delta \mathrm{E}_{\mathrm{j}}\right]=\exp \left[-\Delta \mathrm{E}_{\mathrm{j}} / \mathrm{k} \times \mathrm{T}\right]
$$

When the energy increment is positive, the Monte Carlo process is actually implemented by generating a random number between 0 and 1 . If this random number is $\leq P[\Delta \mathrm{Ej}]$, the particle is moved to the new position; otherwise it remains in its old position.

We have simplified our program by considering the mass of the particles and the temperature to be constant and equal for any particle size and Monte Carlo step. If $\mathrm{m}$ and $\mathrm{T}$ are held constant, the average energy of the system is proportional to the average height of the particles. Therefore, the probability in Eq. [2] can be expressed as:

$$
P\left(\Delta \mathrm{E}_{\mathrm{j}}\right)=\exp \left[-\Delta \mathrm{z}_{\mathrm{j}}(\mathrm{m} \times \mathrm{g} / \mathrm{k} \times \mathrm{T})\right]
$$

where the constant factor $(\mathrm{k} \times \mathrm{T} / \mathrm{m} \times \mathrm{g})$ is the dimensionless temperature of the system.

\section{Packing disk-like particles}

Several computer algorithms that build random packings of spherical particles have 
been developed (Davis and Carter, 1990; Finney, 1975). Rosato et al. (1987) and Barker and Grimson (1990) studied the segregation of disks of various sizes, beginning with a random configuration inside a container and allowing particles to fall to the bottom of the container.

We developed a computer program that packs particles in two dimensions. It is based on the general procedure described by Rosato et al. (1987) whereby particles are situated at random inside a container with periodic boundary conditions perpendicular to the gravity field. The term "periodic boundary conditions" means that those particles that are close to the boundaries of the container, and which would move outside those boundaries when a move is attempted, would re-enter the container through the opposite side. These conditions approximate infinite system simulation without wall effects (Fig. 1a). This procedure allows results from small-systems to be applied to larger systems. Particles are moved according to the Monte Carlo procedure to attain a final configuration where all particles are packed at the bottom of the container.

In our first model, particles can be situated at any location in space inside the container. This places severe demands on computer time as the size of the system increases. Verlet (1967) explains a general computational method to facilitate studying large systems of interacting particles in relation to studying molecular dynamics. The method consists of making a table of all particles that are within a distance of a particle and updating that list after a particle has moved more than a given distance. To increase the speed of the program, we decreased the size of the table as disks were packed in the bottom of the container, because the risk of overlapping was
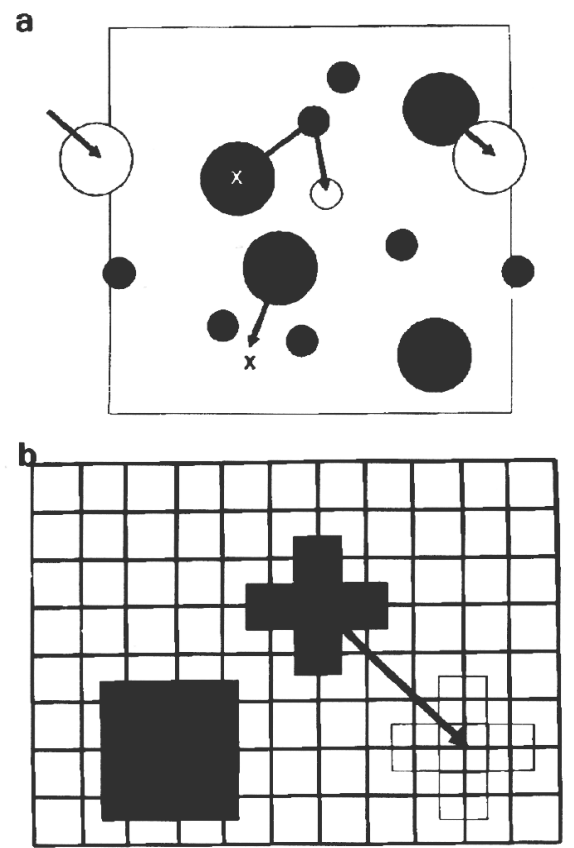

Fig. 1. (a) Continuous and (b) lattice Monte Carlo systems. Arrows indicate attempted moves; $X$ in (a) indicates a blocked move. reduced to a small number of nearest neighbors for each disk. We used FORTRAN 77 and computations were conducted at the central computing facilities of the Univ. of Georgia, using a cluster of IBM RS/6000 model 550 computers.

Particles were packed using two procedures. If we set the dimensionless temperature equal to zero, the probability of any upward move that increases the gravitational potential energy is also zero; therefore, no upward moves are accepted. We call this the "pouring" procedure. We have also implemented a "shaking" procedure, where, using a dimensionless temperature of 0.05 , particles are allowed to move upward according to the probability in Eq. [3].

Our simulations include packing systems of particles of one size (monosize systems) and two sizes (binary systems). Since real container media are three-dimensional systems and have particles of various sizes, the model that we have studied here only demonstrates the potential of Monte Carlo simulations for studying container media. The practical use of Monte Carlo simulation will depend on having more realistic and complex systems. Extending our calculations to such

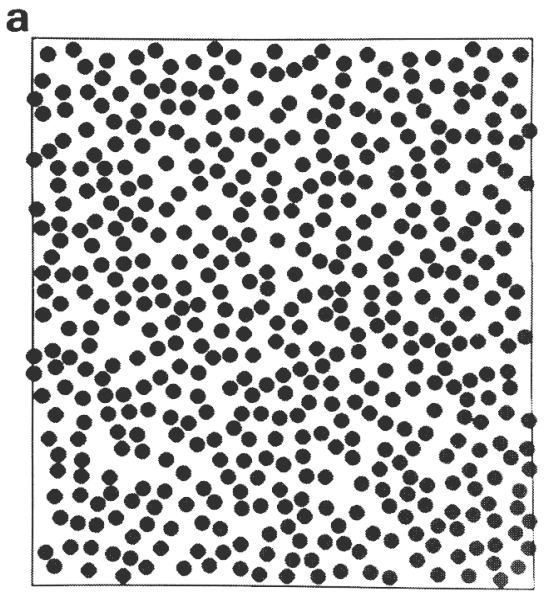

C

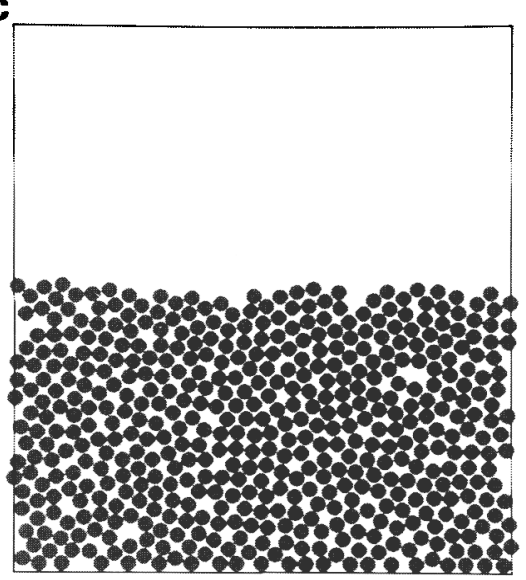

systems is straightforward (Burés et al., 1993).

The porosity was determined from the final distribution of particle positions. Porosity of a two-dimensional system of particles can be expressed as: $\mathrm{P}=$ (total area of the system area covered by particles)/total area of the s y s t e m.

The packing fraction is 1 minus the porosity. Both parameters are usually expressed as percentages. The total area of the system was calculated by implementing an algorithm that computes the average height of the system and the area covered by disks. The porosity of a monosize system was studied, as was the porosity variation when different proportions of disks in binary systems were mixed. Error bars are calculated by repeating the Monte Carlo procedure with various initial configurations and random number sequences. Averages are obtained from 10 runs of the program.

Using lattices to represent various particle shapes

A second approach, which also allows using the Monte Carlo method to introduce various two-dimensional particle shapes, was at-

b

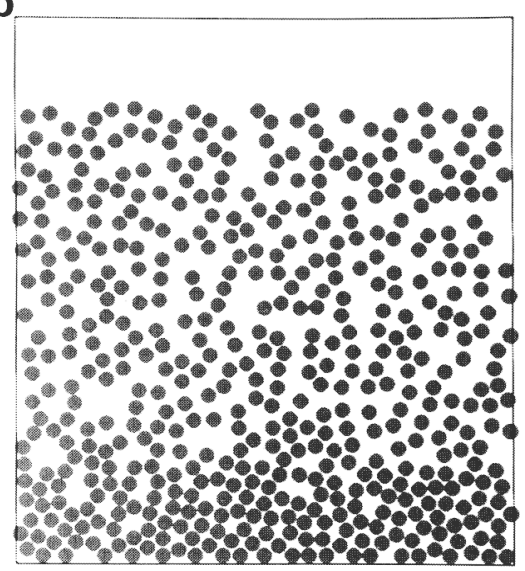

d

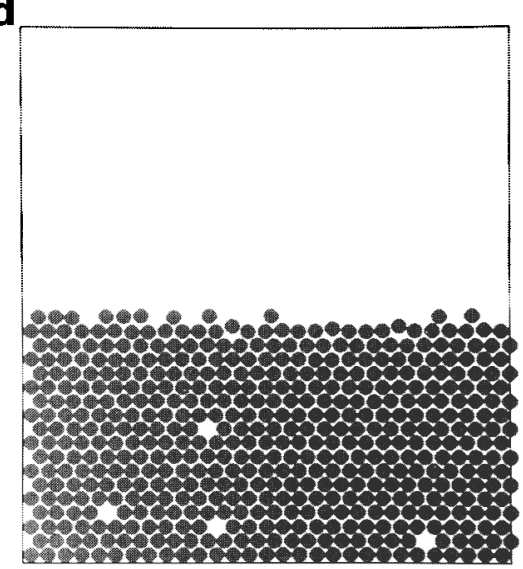

Fig. 2. Monte Carlo simulation of 500 disks (radius $=0.6$ ) with a $35 \times 40$ container size and maximum allowed displacement of 0.1 (dimensionless length units). (a) Initial configuration; (b) configuration after 200 Monte Carlo steps [dimensionless temperature $(\mathrm{T})=0$ ]; (c) randomly packed disks after $10^{6}$ Monte Carlo steps (dimensionless $\mathrm{T}=0$ ); $(\mathbf{d})$ ordered packing after $10^{6}$ Monte Carlo steps (dimensionless $\mathrm{T}=0.05$ ). 
tempted. This approach consists of representing geometrical particles of various sizes and shapes on a lattice (Fig. 1b). Particles are centered at the nodes of the lattice; a given particle can have any shape, depending on the number of nodes that constitute it. Lattice models have been used for efficient programming because they simplify identifying the nearest neighbors of a particle by providing a picture of every site on the lattice (filled or empty) at each time step. Also, the size and shape of pores between particles can be characterized directly by using percolation theory, which deals with identifying neighboring occupied sites (Family and Landau, 1984; Stauffer, 1985).

\section{BEHAVIOR OF SYSTEMS}

Figure 2 shows the time evolution of a 500particle system (each particle's radius $=0.6$ ) inside a $40 \times 35$ container (height $\times$ width) (these should have units of length, but in principle we have taken them as dimensionless). The initial configuration (Fig. 2a), where particles are situated randomly inside the container, serves as the starting point for the Monte Carlo procedure. An attempt is made to move each particle in turn downward. The settling procedure can be seen in Fig. $2 b$, where the system's configuration is represented after 200 Monte Carlo steps. Figures 2 $\mathrm{c}$ and $\mathrm{d}$ show the configurations after 1 million Monte Carlo steps. In Fig. 2c, random packing is obtained for zero dimensionless temperature (particles are allowed to move only downward), while Fig. 2d presents an ordered pattern obtained by rearranging the particles when they are shaken according to the probability in Eq. [3] (dimensionless temperature $=0.05$ ). In Fig. 2d, each particle is in contact with six other particles, as in a dense, ordered packing (close packing). Voids occur where the central particle is missing. This is a consequence of the packing procedure, for once this situation is attained, no particle cart move into the gap.

Packing procedure is of prime importance when a real system is simulated. The configuration obtained after moving particles only downward would simulate "pouring" a soil medium into a container. The porosity of a system thus simulated would resemble that of soil poured into a container and tapped laterally several times to allow particles to resettle (unpublished data). The "shaking" procedure (dimensionless temperature >0) simulates vertically tapping a soil-filled container. We must take this fact into account to avoid introducing external factors in our simulation that would result in discrepancies when comparing our simulated results to those obtained from real systems.

To demonstrate the packing procedure's influence on final results, the evolution of porosity with Monte Carlo time was studied for a system of 1000 monosize particles (Fig. 3 ). A different porosity value was obtained for each of the two packing procedures. Porosities after 1 million Monte Carlo steps are $22.7 \%$ by pouring and $12.3 \%$ by shaking.

Dissimilar packing patterns were obtained,

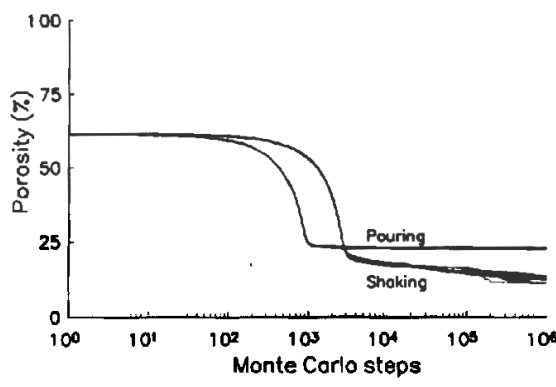

Fig. 3. Porosity (percent) evolution with time (Monte Carlo steps) and packing procedure for a monosize system (radius $=0.6$ ) of 1000 disks. Twopacking procedures have been implemented (pouring and shaking) and 10 replicates have been drawn for each one. Container height is 60 and width 50. Maximum allowed displacement $=0.1$ (dimensionless length units).

depending on the packing procedure. Porosity between spheres is constant for ordered or random packing in three dimensions, but its corresponding value for randomly packed disks in two dimensions seems to be unstable. Berryman (1983) reported an estimate of 0.82 (porosity 18\%) for the packing fraction of dense random packing (shaking particles in- side a container) of disks, and Hinrichsen et al. (1990) gave 0.772 (porosity $22.8 \%$ ) as the estimate for loose random packing (dumping particles into a container). Porosity of a dense ordered packing of disks is $9.3 \%$. Our value for 1000 poured particles agrees with that of Hinrichsen et al. (1990) for loose random packing. Porosity also depends on the system's size. Constant porosity values are obtained for both systems after a certain number of Monte Carlo steps. When particles are only allowed to move downward, equilibrium is reached after 1000 to 5000 Monte Carlo steps, whereas shaking the particles requires more steps to reach equilibrium. The random packing obtained by allowing particles to move downward (Fig. 2c) is a metastable state, where particles cannot change their configuration without introducing a different movement pattern into the simulation. Moving particles upward allows them to rearrange into a stable state. This state also is attained when the starting packing configuration is that of random packing. Figure 3 suggests that by stopping the simulation after a constant number of Monte Carlo steps, we would attain a constant packing pattern. It also allowed us to reduce computer time when obtaining random pack-
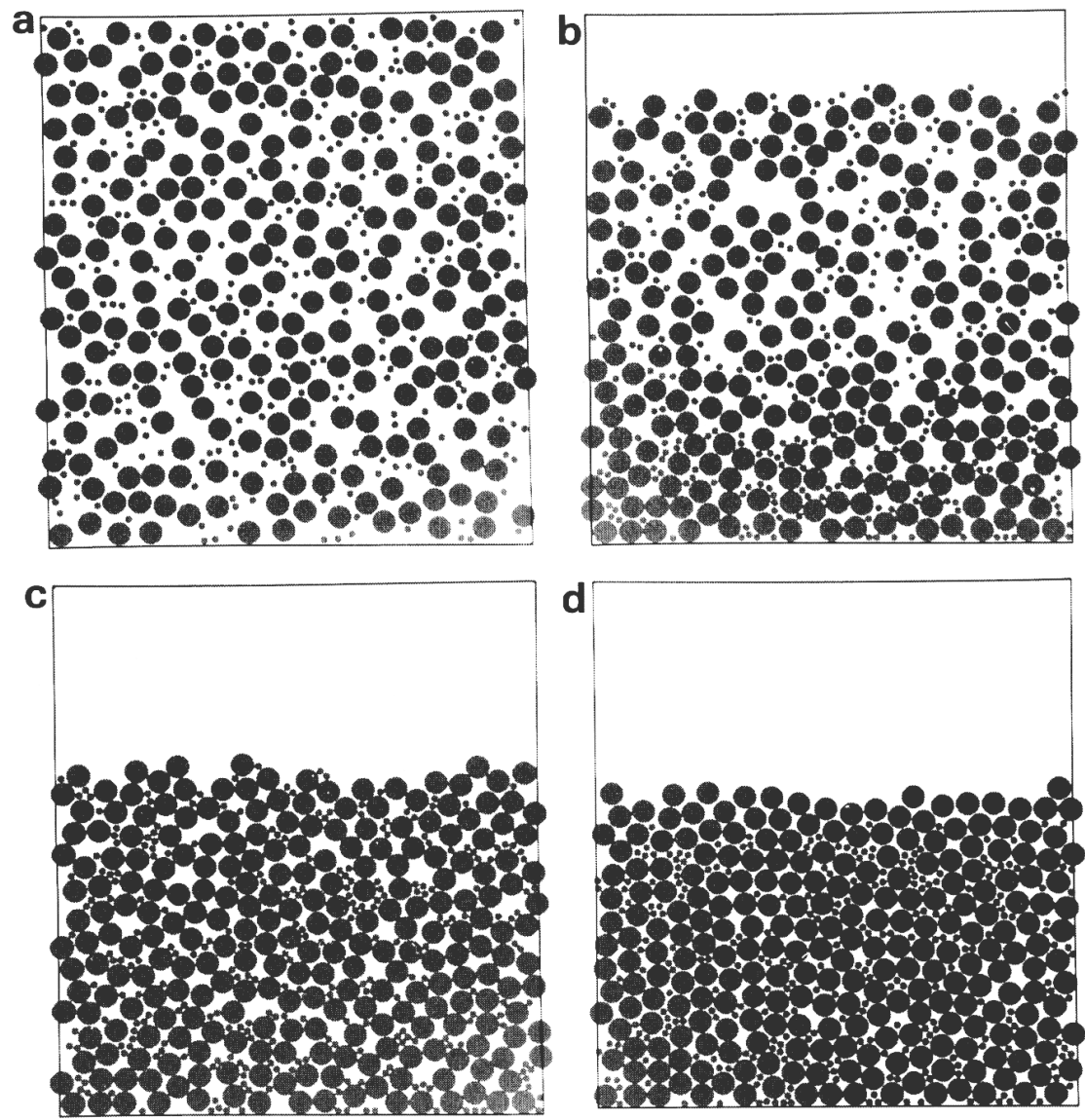

Fig. 4. Monte Carlo simulation of a mixture of 250 disks with radius $=0.9$ and 250 disks with radius $=0.3$ in a $35 \times 40$ container. Maximum allowed displacement $=0.1$ (dimensionless length units). (a) Initial configuration; (b) configuration after 200 Monte Carlo steps [dimensionless temperature (T) $=0$ ]; (c) randomly packed disks after $10^{6}$ Monte Carlo steps (dimensionless $\left.\mathrm{T}=0\right)$; (d) configuration after $10^{6}$ Monte Carlo steps (dimensionless T $=0.05$ ). 
ings. We used 3.5 to 4 min CPU (central processing unit) time for a 1000-disk system and 5000 Monte Carlo steps on an IBM RS/6000.

The simulation process was repeated for a binary system of particles (Fig. 4). Final equilibrium, when shaking this system, was not reached even after 1 million Monte Carlo steps. According to Rosato et al. (1987), shaking would result in particle segregation, where large particles rise above the small ones. A certain amount of segregation can be seen in Fig. 4d. Clustering of small particles between large particles is a phenomenon that takes place in actual soils and container media. We have observed this clustering phenomenon in our simulation (Fig. 4c). Clustering occurs when a small particle situated above two large particles moves into a gap between the larger particles, thus preventing the two large particles from moving toward each other. The small particles, in being pushed downward, maintain the gap open for an incoming small particle, resulting in a chain-like process. This phenomenon, we believe, could be useful in studying several soil processes, such as organic matter decay, particle breakdown, nutrient movement, or erosion. For the configuration in Fig. 4c, it also would be possible to choose several particles randomly and change their size with time, or break them into smaller particles. This procedure would allow the study of various soil phenomena, thus opening many possibilities for simulation if actual relationships between real soils and the simulated system are established. Many variables have to be considered: particle shape, packing procedure, number of spheres and container size, actual distribution of particle sizes, and composition and surface properties of the soil particles.

Porosity evolution by mixing two particle sizes also has been studied (Fig. 5a). Visscher and Bolsterli (1972) proposed simulating this phenomenon via a Monte Carlo procedure. Bodman and Constantin (1965), Sohn and Moreland (1968), and Westman (1936) have published experimental results. Porosity (as a percentage) of monosize systems has a constant value and does not depend on particle size. When two sizes are mixed, porosity decreases to a minimum value. This volume shrinkage on mixing different particle sizes leads to soil compaction (Bodman and Constantin, 1965) and is important in relation to container media, considering that commercial media generally consist of mixtures of several materials with dissimilar particle size distributions. For a small number of particles, porosity also depends on systemize. In studying this phenomenon via simulation, we used two starting systems that had the same area covered by particles-1000 large particles (radius $=0.9$ ) and 9000 small particles (radius $=0.3$ ) - and we poured them for 5000 Monte Carlo steps. Porosity depends on the number of particles for small systems (Figs. 5b and $5 \mathrm{c})$, which explains the asymmetry at the extremes of Fig. 5a.

Figure 6 shows a simulated lattice system, Particles of two sizes and shapes were settled inside a container by Monte Carlo procedure.
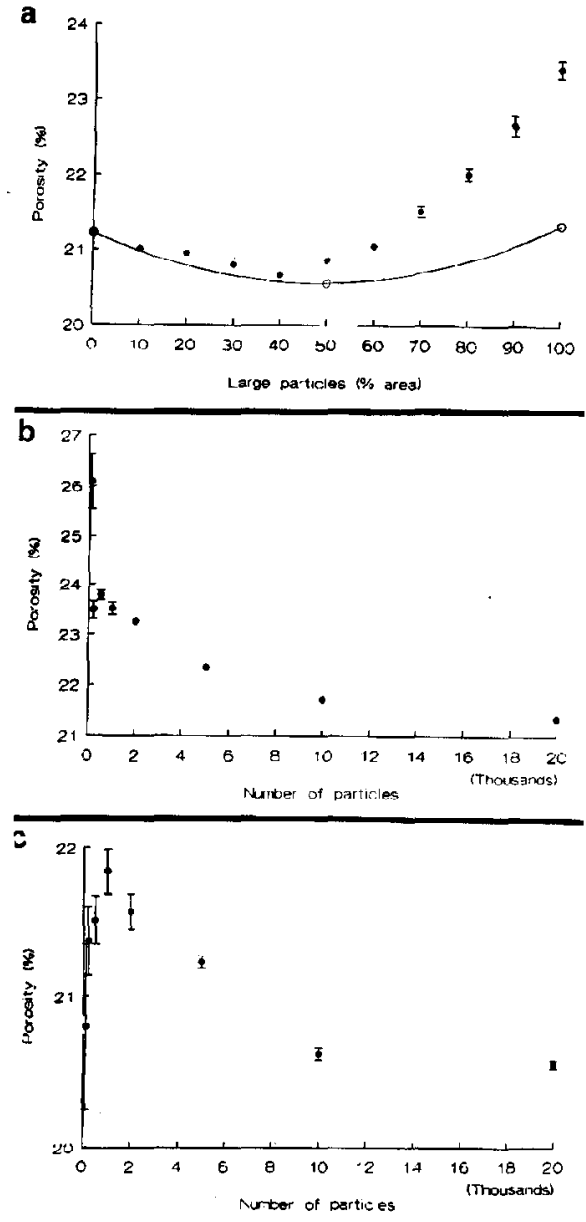

Fig. 5. (a) Dependence of porosity (percent) of a binary mixture of disks (large-paricle radius = 0.9 ; small-particle radius $=0.3$ ) on the percentage of large disks in the mixture. Pouring time equals 5000 Monte Carlo steps. All abscissa points have the same particle density ( 2544.69 square units, equivalent to 1000 large disks and 9000 small disks). Solid line indicates theoretical curve with no influence of particle number. Dependence of porosity after 5000 Monte Carlo steps on the number of particles in a (b) monosize system and (c) binary system with the same area of large and small particles.

Although this can be considered a simplistic model, it has a major advantage over the previous model of spherical particles in that any particle shape can be represented by increasing the number of lattice sites that constitute a single particle. In our case, crosses consist of five lattice sites (see Fig. 1) and squares of nine lattice sites. Also, pore size can be easily determined by counting the number of lattice sites that are trapped between the particles. Although our results with this system are still preliminary, we foresee many possibilities in characterizing water movement, by assessing which pores are connected, and in determining pore size distribution. Nutrient and pesticide leaching could be evaluated by Monte Carlo techniques, which could be incorporated into studies of ground water pollution.

Monte Carlo simulation can help us build models for characterizing horticultural systems, not only in studying physical systems

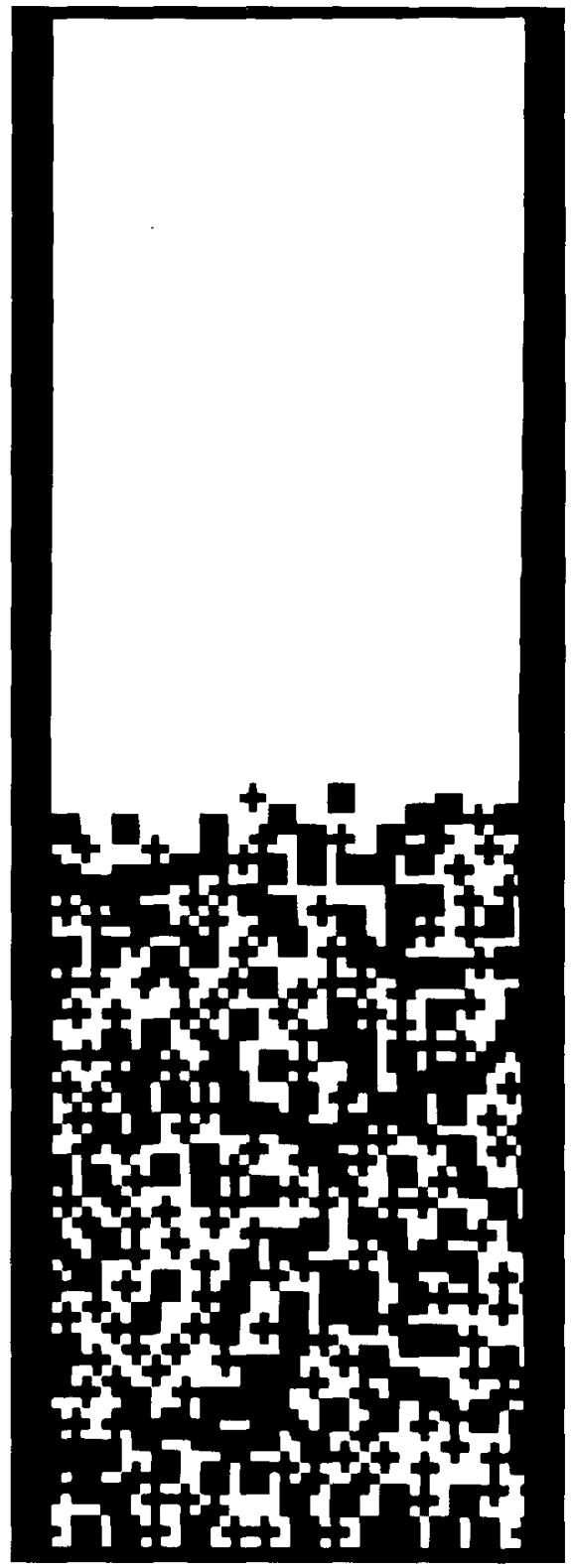

Fig. 6. Lattice system of 150 particles of each type after 5000 Monte Carlo steps at dimensionless temperature $=0.05$. Lattice size is $50 \times 150$ lattice sites. Maximum allowed displacement = five lattice sites.

that support plant growth, but also in developing applications for plant growth itself. Approaching tissue development as a random growth process would allow using the Monte Carlo technique. Root growth patterns influenced by type of medium particles and container configuration is another application for Monte Carlo simulation in horticulture. Water and nutrient uptake by plants also could be studied using Monte Carlo simulation.

Incorporating basic techniques that have been developed in other areas-in our case, physics-into horticultural research can provide useful information for understanding the biological systems involved. We believe that interdisciplinary research can serve this purpose. This could require learning concepts that may not be commonly used in horticulture but that will certainly help horticultural researchers keep pace with modern developments. 


\section{Literature Cited}

Barker, G. and M. Grimson. 1990. The physics of muesli. New Scientist 126(1718):37-40.

Berryman, J.G. 1983. Definition of dense random packing, p. 1-18. In: M. Shahinpoor (ed.). Advances in the mechanics and the flow of granular materials. Gulf, Houston.

Binder, K. 1986. Introduction Theory and "technical" aspects of Monte Carlo simulations, p. 145. In: K. Binder (ed.). Monte Carlo methods in statistical physics. 2nd ed. Springer-Verlag, Berlin.

Bodman, G.B and G.K. Constantine. 1965. Influence of particle size distribution in soil compaction. Hilgardia 35:567-591.

Bugbee, G.J. and C.R. Frink. 1983. Quality of potting soils. Connecticut Agr. Expt. Sta. Bul. 812.

Burés, S., F.X. Martinez, and M. Llorca. 1988. Preliminary study of the application of parametric linear programming in formulation of substrate mixes. Acta Hort. 221:141-152.

Burés, S., F.A. Pokorny, D.P. Landau, and A.M. Ferrenberg. 1993. Computer simulation of volume shrinkage upon mixing container media components. J. Amer. Soc. Hort. Sci. 118:757761.

Dallavalle, J.M. 1948. Micromeritics. 2nd. ed. Pitman Publishing, London.

Davis, I.L. and R.G. Carter. 1990. Random particle packing by reduced dimension algorithms. J. Applied Physics 67:1022-1029.

Family, F. and D.P. Landau. 1984. Kinetics of aggregation and gelation. North-Holland Physics. Netherlands.
Ferrenberg, A.M. and D.P. Landau. 1991. Critical behavior of the three-dimensional Ising model. A high-resolution Monte Carlo study. Phys. Rev. B 44:5081-5091.

Finney, J.L. 1975. The structures of laboratory and computer-built random packing. J. Physique (Paris)36:C2-1-11.

Finney, J.L. 1983. Structure and properties of granular materials: Guidelines from modelling studies of liquids and amorphous solids, p. 19-39. In: M. Shahinpoor (ed.). Advances in the mechanics and the flow of granular materials. Gulf, Houston.

Gould, H. and J. Tobochnik. 1988. An introduction to computer simulation methods. Applications to physical systems. Addison-Wesley, Reading, Mass.

Hammersley, J.M. and D.C. Handscomb. 1964 Monte Carlo methods. Spottiswonde, Ballantine, and Co., London.

Hinrichsen, E.L., J. Feder, and T. Jossang. 1990. Random packing of disks in two dimensions. Phys. Rev. A. 41:4199-4209.

Landau, D.P. and R. Alben. 1973. Monte Carlo calculations as an aid in teaching statistical mechanics. Amer. J. Physics 41:394-430.

Metropolis, N., A.W. Rosenbluth, M.N. Rosenbluth, A.H. Teller, and E. Teller. 1953. Equation of state calculations by fast computing machines. J. Chem. Physics 21:1087-1092.

Milks, R.R., W.C. Fonteno, and R.A. Larson. 1989. Hydrology of horticultural substrates: II. Predicting physical properties of media in containers. J. Amer. Soc. Hort. Sci. 114:53-56.

Penninck, R., O. Verdonck, and M. De Boodt. 1985.
Different materials which can be used in composts. Acta Hort. 172:31-38.

Pokorny, F.A. 1981. Rx media-A concept for container plant production. Amer. Soc. Agr. Eng. paper 81-1089. St. Joseph, Mich.

Rosato, A., K.J. Strandburg, F. Prinz, and R.H. Swendsen. 1987. Why the Brazil nuts are on top: Size segregation of particulate matter by shaking. Phys. Rev. Lett. Amer. Phys. Soc. 58:10381040.

Slichter, C.S. 1899. Theoretical investigation of the motion of ground waters. 19th Annu. Rpt. U.S. Geological Survey, Washington, D.C. Government Printing Office 19:301-384.

Sohn, H.Y. and C. Moreland. 1968. The effect of particle size distribution on packing density. Can. J. Chem. Eng. 46:162-167.

Stauffer, D. 1985. Introduction to percolation theory. Taylor and Francis, London.

Tilt, K.M., T.E. Bilderback, and W.C. Fonteno. 1987. Particle size and container size effects on growth of three ornamental species. J. Amer. Soc. Hort. Sci. 112:981-984.

Verlet, L. 1967. Computer "experiments" on classical fluids. I. Thermodynamical properties of Lennard-Jones molecules. Phys. Rev. 159:98103.

Visscher, W.M. and M. Bolsterli. 1972. Random packing of equal and unequal spheres in two and three dimensions. Nature (London) 239:504507.

Westman, A.E.R. 1936. The packing of particles: Empirical equations for intermediate diameter ratios. Amer. Ceramic Soc. J. 19:127-129. 